\title{
Recent results from the Belle experiment
}

\author{
Dmitri Liventsev ${ }^{1,2, \star, \star \star}$ \\ ${ }^{1}$ Virginia Polytechnic Institute and State University, Blacksburg, Virginia 24061 \\ ${ }^{2}$ High Energy Accelerator Research Organization (KEK), Tsukuba 305-0801
}

\begin{abstract}
We review recent results obtained using the data recorded with the Belle detector at the KEKB asymmetric-energy $e^{+} e^{-}$collider in KEK, Japan.
\end{abstract}

\section{Introduction}

The Belle detector is a large-solid-angle magnetic spectrometer that consists of a silicon vertex detector (SVD), a 50-layer central drift chamber (CDC), an array of aerogel threshold Cherenkov counters (ACC), a barrel-like arrangement of time-of-flight scintillation counters (TOF), and an electromagnetic calorimeter comprised of $\mathrm{CsI}(\mathrm{Tl})$ crystals (ECL) located inside a super-conducting solenoid coil that provides a $1.5 \mathrm{~T}$ magnetic field. An iron flux-return located outside of the coil is instrumented to detect $K_{L}^{0}$ mesons and to identify muons (KLM). The detector is described in detail elsewhere [1].

The Belle experiment successfully operated for more than a decade until 2010 at the asymmetricenergy $e^{+} e^{-}$collider KEKB [2] in various $\Upsilon(n S)$ resonances, having collected a world-record sample of data over $1 \mathrm{ab}^{-1}$.

Here we present a review of recent results from Belle based on its full statistics.

\section{Angular Analysis of $B^{0} \rightarrow K^{*}(892)^{0} \ell^{+} \ell^{-}$}

Rare decays of $B$ mesons are an ideal probe to search beyond the Standard Model (SM) of particle physics, since contributions from new particles lead to effects that are of similar size as the SM predictions. The rare decay $B^{0} \rightarrow K^{*}(892)^{0} \ell^{+} \ell^{-}$, where $\ell^{+} \ell^{-}$is either $e^{+} e^{-}$or $\mu^{+} \mu^{-}$, involves the quark transition $b \rightarrow s \ell^{+} \ell^{-}$, a flavor changing neutral current that is forbidden at tree level in the SM. Higher order SM processes such as penguin or $W^{+} W^{-}$box diagrams allow for such transitions, leading to branching fractions of less than one in a million. Various extensions to the SM predict contributions from new physics, which can interfere with the SM amplitudes and lead to enhanced or suppressed branching fractions or modified angular distributions of the decay products.

Belle presented an angular analysis [3], using the decay modes $B^{0} \rightarrow K^{*}(892)^{0} e^{+} e^{-}$and $B^{0} \rightarrow$ $K^{*}(892)^{0} \mu^{+} \mu^{-}$, in a data sample recorded with the Belle detector. The LHCb collaboration reported a discrepancy in the angular distribution of the decay $B^{0} \rightarrow K^{*}(892)^{0} \mu^{+} \mu^{-}$, corresponding to a $3.4 \sigma$ deviation from the SM prediction [4]. In contrast to the $\mathrm{LHCb}$ measurement the di-electron channel is also used in this analysis.

$\star$ e-mail: dmitri.liventsev@kek.jp

$\star \star$ On behalf of the Belle collaboration 
Table 1. Fitted yields and statistical error for signal $\left(n_{\text {sig }}\right)$ and background $\left(n_{\text {bkg }}\right)$ events in the binning of $q^{2}$ for both the combined electron and muon channel.

\begin{tabular}{cccc}
\hline \hline Bin & $q^{2}$ range in $\mathrm{GeV}^{2} / c^{4}$ & $n_{\text {sig }}$ & $n_{\text {bkg }}$ \\
\hline 0 & $1.00-6.00$ & $49.5 \pm 8.4$ & $30.3 \pm 5.5$ \\
\hline 1 & $0.10-4.00$ & $30.9 \pm 7.4$ & $26.4 \pm 5.1$ \\
2 & $4.00-8.00$ & $49.8 \pm 9.3$ & $35.6 \pm 6.0$ \\
3 & $10.09-12.90$ & $39.6 \pm 8.0$ & $19.3 \pm 4.4$ \\
4 & $14.18-19.00$ & $56.5 \pm 8.7$ & $16.0 \pm 4.0$ \\
\hline \hline
\end{tabular}

$K^{*}$ candidates are formed in the channel $K^{* 0} \rightarrow K^{+} \pi^{-}$and are combined with oppositely charged lepton pairs to form $B$ meson candidates. The large combinatoric background is suppressed by applying requirements on kinematic variables. Two independent variables can be constructed using constraints that in $\Upsilon(4 S)$ decays $B$ mesons are produced pairwise and each carries half the centerof-mass $(\mathrm{CM})$ frame beam energy, $E_{\mathrm{Beam}}$. These variables are the beam constrained mass, $M_{\mathrm{bc}}$, and the energy difference, $\Delta E$, in which signal features a distinct distribution that can discriminate against background. The variables are defined in the $\Upsilon(4 S)$ rest frame as

$$
\begin{aligned}
M_{\mathrm{bc}} & \equiv \sqrt{E_{\mathrm{Beam}}^{2} / c^{4}-\left|\vec{p}_{B}\right|^{2} / c^{2}} \text { and } \\
\Delta E & \equiv E_{B}-E_{\mathrm{Beam}},
\end{aligned}
$$

where $E_{B}$ and $\left|\vec{p}_{B}\right|$ are the energy and momentum of the reconstructed candidate, respectively. Correctly reconstructed candidates are located around the nominal $B$ mass in $M_{\mathrm{bc}}$ and feature $\Delta E$ of around zero. Candidates are selected satisfying $5.22<M_{\mathrm{bc}}<5.3 \mathrm{GeV} / c^{2}$ and $-0.10(-0.05)<$ $\Delta E<0.05 \mathrm{GeV}$ for $\ell=e(\ell=\mu)$.

Large irreducible background contributions arise from charmonium decays $B \rightarrow K^{(*)} J / \psi$ and $B \rightarrow K^{(*)} \psi(2 S)$, in which the $c \bar{c}$ state decays into two leptons. These decays have the same signature as the desired signal and are vetoed with the following requirements on $q^{2}=M_{\ell^{+} \ell^{-}}$, the invariant mass of the lepton pair: $-0.25 \mathrm{GeV} / c^{2}<M_{e e(\gamma)}-m_{J / \psi}<0.08 \mathrm{GeV} / c^{2},-0.15 \mathrm{GeV} / c^{2}<M_{\mu \mu}-m_{J / \psi}<$ $0.08 \mathrm{GeV} / c^{2},-0.20 \mathrm{GeV} / c^{2}<M_{e e(\gamma)}-m_{\psi(2 S)}<0.08 \mathrm{GeV} / c^{2}$ and $-0.10 \mathrm{GeV} / c^{2}<M_{\mu \mu}-m_{\psi(2 S)}<$ $0.08 \mathrm{GeV} / c^{2}$.

For the angular analysis the number of signal events $n_{\text {sig }}$ and background events $n_{\text {bkg }}$ in the signal region $M_{\mathrm{bc}}>5.27 \mathrm{GeV} / c^{2}$ are obtained by a fit to $M_{\mathrm{bc}}$ in bins of $q^{2}$. The extracted yields and the definition of the bin ranges are presented in Table 1.

We perform an angular analysis of $B^{0} \rightarrow K^{*}(892)^{0} \ell^{+} \ell^{-}$including the electron and muon modes. The decay is kinematically described by three angles $\theta_{\ell}, \theta_{K}$ and $\phi$ and the invariant mass squared of the lepton pair $q^{2}$. Definitions of the angles and the full angular distribution follow Ref. [5]. The binning in $q^{2}$ is detailed in Table 1 together with the measured signal and background yields. Uncovered regions in the $q^{2}$ spectrum arise from vetoes against backgrounds of the charmonium resonances $J / \psi \rightarrow \ell^{+} \ell^{-}$and $\psi(2 S) \rightarrow \ell^{+} \ell^{-}$and vetos against $\pi^{0}$ Dalitz decays and photon conversion.

The observables $P_{i=4,5,6,8}^{\prime}$, introduced in Ref. [6] are functions of Wilson coefficients, containing information about the short-distance effects and can be affected by new physics and are considered to be largely free from form-factor uncertainties [7]. The statistics in this analysis are not sufficient to perform an eight-dimensional fit, therefore a folding technique is used explained in more detail in Refs. [8].

The signal and background fractions are derived from a fit to beforehand, where the yields are listed in Table 1. The $M_{\mathrm{bc}}$ variable is split into a signal (upper) and sideband (lower) region at 
$5.27 \mathrm{GeV} / c^{2}$. In the second step the shape of the background for the angular observables is estimated on the $M_{\mathrm{bc}}$ sideband. This is possible as the angular observables have shown to be uncorrelated to $M_{\mathrm{bc}}$ in the background sample.

All observables $P_{i=4,5,6,8}^{\prime}$ are extracted from the data in the signal region using three-dimensional unbinned maximum likelihood fits in four bins of $q^{2}$ and the additional zeroth bin using the folded signal PDF, fixed background shapes and a fixed number of signal events. Each $P_{i=4,5,6,8}^{\prime}$ is fitted with the $K^{*}$ longitudinal polarization $F_{L}$ and the transverse polarization asymmetry $A_{T}^{(2)}$. Counting also the zeroth bin, which exhibits overlap with the range of the first and second bin, 20 measurements are performed.

The measurements are compared with SM predictions based upon different theoretical calculations. The result for $P_{5}^{\prime}$ is shown in Fig. 1 together with available SM prediction and LHCb measurements. A deviation with respect to the SM prediction is observed with a significance of $2.1 \sigma$ in the $q^{2}$ range $4.0<q^{2}<8.0 \mathrm{GeV}^{2} / c^{4}$. This deviation is into the same direction and in the same $q^{2}$ region where the LHCb collaboration reported the so-called $P_{5}^{\prime}$ anomaly $[4,8]$.

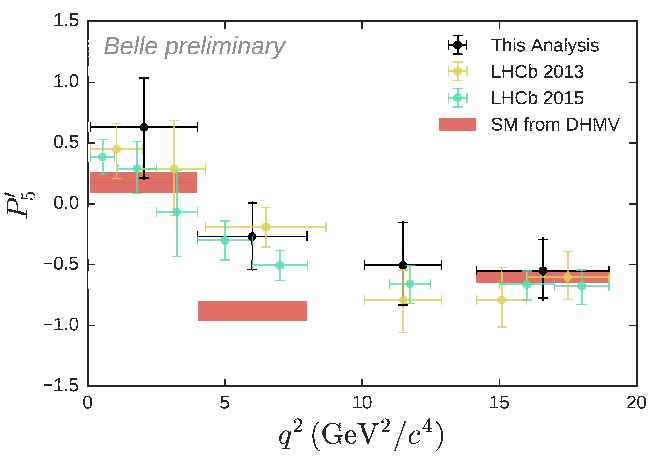

Figure 1. Result for the $P_{5}^{\prime}$ observable compared to SM predictions from various sources. Results from $\mathrm{LHCb}$ $[4,8]$ are shown for comparison.

\section{Measurement of the branching ratio of $\bar{B}^{0} \rightarrow D^{*+} \tau^{-} \bar{\nu}_{\tau}$}

Semitauonic $B$ meson decays of the type $b \rightarrow c \tau v_{\tau}$ are sensitive probes to search for physics beyond the Standard Model (SM). Charged Higgs bosons, which appear in supersymmetry and other models with at least two Higgs doublets, may contribute to the decay to due to large mass of the $\tau$ lepton and induce measurable effects in the branching fraction. Similarly, leptoquarks, which carry both baryon number and lepton number, may also contribute to this process. The ratio of branching fractions

$$
\mathcal{R}\left(D^{(*)}\right)=\frac{\mathcal{B}\left(\bar{B} \rightarrow D^{(*)} \tau^{-} \bar{v}_{\tau}\right)}{\mathcal{B}\left(\bar{B} \rightarrow D^{(*)} \ell^{-} \bar{v}_{\ell}\right)} \quad(\ell=e, \mu)
$$

is typically used instead of the absolute branching fraction of $\bar{B} \rightarrow D^{(*)+} \tau^{-} \bar{v}_{\tau}$, to reduce several systematic uncertainties such as those on the experimental efficiency, the CKM matrix elements $\left|V_{c b}\right|$, and on the form factors. The SM calculations on these ratios predict $\mathcal{R}\left(D^{*}\right)=0.252 \pm 0.003$ [9] and $\mathcal{R}(D)=0.297 \pm 0.017[10,14]$ with precision of better than $2 \%$ and $6 \%$ for $\mathcal{R}\left(D^{*}\right)$ and $\mathcal{R}(D)$, respectively. Exclusive semitauonic $B$ decays were first observed by the Belle Collaboration [12], with subsequent studies reported by Belle [13, 14], BABAR [11], and LHCb [15] Collaborations. All 
results are consistent with each other, and the average values of Refs. [11, 14, 15] have been found to be $\mathcal{R}\left(D^{*}\right)=0.322 \pm 0.018 \pm 0.012$ and $\mathcal{R}(D)=0.391 \pm 0.041 \pm 0.028$ [16], which exceed the SM predictions for $\mathcal{R}\left(D^{*}\right)$ and $\mathcal{R}(D)$ by $3.0 \sigma$ and $1.7 \sigma$, respectively. The combined analysis of $\mathcal{R}\left(D^{*}\right)$ and $\mathcal{R}(D)$, taking into account measurement correlations, finds that the deviation is $3.9 \sigma$ from the SM prediction.

In the paper [17] Belle reported the first measurement of $\mathcal{R}\left(D^{*}\right)$ using the semileptonic tagging method. Signal $B^{0} \bar{B}^{0}$ events are reconstructed in modes where one $B$ decays semi-tauonically $\bar{B}^{0} \rightarrow D^{*+} \tau^{-} \bar{v}_{\tau}$ where $\tau^{-} \rightarrow \ell^{-} \bar{v}_{\ell} v_{\tau}$, (referred to hereafter as $B_{\text {sig }}$ ) and the the other $B$ decays in a semileptonic channel $\bar{B}^{0} \rightarrow D^{*+} \ell^{-} \bar{v}_{\ell}$ (referred to hereafter as $B_{\text {tag }}$ ). To reconstruct normalization $B^{0} \bar{B}^{0}$ events, which correspond to the denominator in $\mathcal{R}\left(D^{*}\right)$, both $B$ mesons are reconstructed decaying to semileptonic decay modes $D^{* \pm} \ell^{\mp} \bar{v}_{\ell}$.

To tag semileptonic $B$ decays, we combine $D^{*+}$ meson and lepton candidates of opposite electric charge and calculate the cosine of the angle between the momentum of the $B$ meson and the $D^{*} \ell$ system in the $\Upsilon(4 S)$ rest frame, under the assumption that only one massless particle is not reconstructed:

$$
\cos \theta_{B-D^{*} \ell} \equiv \frac{2 E_{\mathrm{beam}} E_{D^{*} \ell}-m_{B}^{2}-M_{D^{*} \ell}^{2}}{2\left|\vec{p}_{B}\right| \cdot\left|\vec{p}_{D^{*} \ell}\right|}
$$

where $E_{\text {beam }}$ is the energy of the beam, and $E_{D^{*} \ell}, \vec{p}_{D^{*} \ell}$ and $M_{D^{*} \ell}$ are the energy, momentum, and mass of the $D^{*} \ell$ system, respectively. The variable $m_{B}$ is the nominal $B$ meson mass [18], and $\vec{p}_{B}$ is the nominal $B$ meson momentum. All variables are defined in the $\Upsilon(4 S)$ rest frame. Correctly reconstructed $B$ candidates in the tag and normalization mode $D^{*} \ell v_{\ell}$ are expected to have a value of $\cos \theta_{B-D^{*} \ell}$ between -1 and +1 . On the other hand, correctly reconstructed $B$ candidates in the signal decay mode $D^{*} \tau v_{\tau}$ or falsely reconstructed $B$ candidates would tend to have values of $\cos \theta_{B-D^{*} \ell}$ below the physical region due to contributions from additional particles and a large negative correlation with missing mass squared, $M_{\text {miss }}^{2}=\left(2 E_{\text {beam }}-\sum_{i} E_{i}\right)^{2} / c^{4}-\left|\sum_{i} \vec{p}_{i}\right|^{2} / c^{2}$, where $\left(\vec{p}_{i}, E_{i}\right)$ is four-momentum of the particles in the $\Upsilon(4 S)$ rest frame.

In each event we require two tagged $B$ candidates that are opposite in flavor. Signal events may have the same flavor due to the $B \bar{B}$ mixing, however we veto such events as they lead to ambiguous $D^{*} \ell$ pair assignment and larger combinatorial background. We require that at most one $B$ meson is reconstructed in a $D^{+}$mode, in order to avoid large background from fake neutral pions when forming $D^{*}$ candidates. In each signal event we assign the candidate with the lowest value of $\cos \theta_{B-D^{*} \ell}$ (referred to hereafter as $\cos \theta_{B-D^{*} \ell}^{\text {sig }}$ ) as $B_{\text {sig. }}$.

To separate reconstructed signal and normalization events, we employ a neural network approach. The variables used as inputs to the network are (i) $\cos \theta_{B-D^{*} \ell}^{\text {sig }}$, (ii) missing mass squared, $M_{\text {miss }}^{2}$, and (iii) visible energy $E_{\mathrm{vis}}=\sum_{i} E_{i}$, where $E_{i}$ is energies of the particles in the $\Upsilon(4 S)$ rest frame. To separate signal and normalization events from background processes, we use the extra energy, $E_{\mathrm{ECL}}$, which is defined as the sum of the energies of neutral clusters detected in the ECL that are not associated with reconstructed particles.

We extract the signal and normalization yields using a two-dimensional extended maximumlikelihood fit in $N N$ and $E_{\mathrm{ECL}}$. The projection of the fitted distributions are shown in Figure 2. The yields of signal and normalization events are measured to be $231 \pm 23$ (stat) and $2800 \pm 57$ (stat), respectively. The ratio $\mathcal{R}\left(D^{*}\right)$ is therefore found to be

$$
\mathcal{R}\left(D^{*}\right)=0.302 \pm 0.030 \pm 0.011
$$

where the first and second errors correspond to statistical and systematic uncertainties, respectively.

We calculate the statistical significance of the signal as $\sqrt{-2 \ln \left(\mathcal{L}_{0} / \mathcal{L}_{\max }\right)}$, where $\mathcal{L}_{\max }$ and $\mathcal{L}_{0}$ are the maximum likelihood and the likelihood obtained assuming zero signal yield, respectively. We 


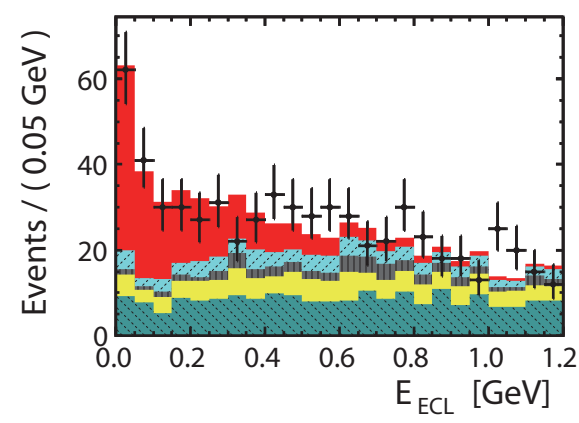

Figure 2. Projections of the fit results with data points overlaid of $E_{\mathrm{ECL}}$ distribution with signal-enhanced $N N$ region $(N N>0.8$. The background categories are described in detail in the text, where "others" refers to predominantly $B \rightarrow X_{c} D^{*}$ decays.

obtain a statistical significance of $13.8 \sigma$. We also estimate the compatibility of the measured value of $\mathcal{R}\left(D^{*}\right)$ and the SM prediction. The effect of systematic uncertainties are included by convolving the likelihood function with a Gaussian distribution. We obtain that our result is larger than the SM prediction by $1.6 \sigma$.

We investigated the compatibility of the data samples with type II two-Higgs-doublet model (2HDM) and leptoquark models. We find our data is compatible with the SM and type II 2HDM with $\tan \beta / m_{H^{+}}=0.7 \mathrm{GeV}^{-1}$, while the $R_{2}$ type leptoquark model with $C_{T}=+0.36$ is disfavored.

\section{Observation of the decay $B_{s}^{0} \rightarrow K^{0} \bar{K}^{0}$}

The two-body decays $B_{s}^{0} \rightarrow h^{+} h^{-}$, where $h^{(\prime)}$ is either a pion or kaon, have now all been observed [18]. In contrast, the neutral-daughter decays $B_{s}^{0} \rightarrow h^{0} h^{\prime 0}$ have yet to be observed. The decay $B_{s}^{0} \rightarrow K^{0} K^{0}$ is of particular interest because the branching fraction is predicted to be relatively large. In the Standard model, the decay proceeds mainly via a $b \rightarrow s$ loop (or "penguin") transition and the branching fraction is predicted to be in the range $(16-27) \times 10^{-6}$ [20]. The presence of non-SM particles or couplings could enhance this value [21]. It has been pointed out that $C P$ asymmetries in $B_{s}^{0} \rightarrow K^{0} \bar{K}^{0}$ decays are promising observables in which to search for new physics [22].

The current upper limit on the branching fraction, $\mathcal{B}\left(B_{s}^{0} \rightarrow K^{0} \bar{K}^{0}\right)<6.6 \times 10^{-5}$ at $90 \%$ confidence level, was set by the Belle Collaboration using $23.6 \mathrm{fb}^{-1}$ of data recorded at the $\Upsilon(5 S)$ resonance [23]. In paper [19] Belle updates this result using the full data set of $121.4 \mathrm{fb}^{-1}$ recorded at the $\Upsilon(5 S)$. The analysis presented here uses improved tracking, $K^{0}$ reconstruction, and continuum suppression algorithms. The data set corresponds to $(6.53 \pm 0.66) \times 10^{6} B_{s}^{0} \bar{B}_{s}^{0}$ pairs [24] produced in three $\Upsilon(5 S)$ decay channels: $B_{s}^{0} \bar{B}_{s}^{0}, B_{s}^{* 0} \overline{B_{s}^{0}}$ or $B_{s}^{0} \bar{B}_{s}^{* 0}$, and $B_{0}^{* 0} \bar{B}_{s}^{* 0}$. The latter two channels dominate, with production fractions of $f_{B_{s}^{* 0} \bar{B}_{s}^{0}}=(7.3 \pm 1.4) \%$ and $f_{B_{s}^{* 0} \bar{B}_{s}^{* 0}}=(87.0 \pm 1.7) \%[25]$.

Candidate $K^{0}$ mesons are reconstructed via the decay $K_{s} \rightarrow \pi^{+} \pi^{-}$using a neural network (NN) technique. To identify $B_{s}^{0} \rightarrow K_{s} K_{s}$ candidates, we define two variables: the beam-energy-constrained mass $M_{\mathrm{bc}}=\sqrt{E_{\text {beam }}^{2}-\left|\vec{p}_{B}\right|^{2} c^{2}} / c^{2}$; and the energy difference $\Delta E=E_{B}-E_{\text {beam }}$, where $E_{\text {beam }}$ is the beam energy and $E_{B}$ and $\vec{p}_{B}$ are the energy and momentum, respectively, of the $B_{S}^{0}$ candidate. These quantities are evaluated in the $e^{+} e^{-}$center-of-mass frame. We require that events satisfy $M_{\mathrm{bc}}>5.34 \mathrm{GeV} / c^{2}$ and $-0.20 \mathrm{GeV}<\Delta E<0.10 \mathrm{GeV}$. To suppress background arising from 
continuum $e^{+} e^{-} \rightarrow q \bar{q}(q=u, d, s, c)$ production, we use a second NN that distinguishes jetlike continuum events from more spherical $B_{s}^{(*) 0} \bar{B}_{s}^{(*) 0}$ events. The NN has a single output variable $\left(C_{\mathrm{NN}}\right)$ that ranges from -1 for backgroundlike events to +1 for signal-like events. We require $C_{\mathrm{NN}}>-0.1$, which rejects approximately $85 \%$ of $q \bar{q}$ background while retaining $83 \%$ of signal decays. We subsequently translate $C_{\mathrm{NN}}$ to a new variable

$$
C_{\mathrm{NN}}^{\prime}=\ln \left(\frac{C_{\mathrm{NN}}-C_{\mathrm{NN}}^{\min }}{C_{\mathrm{NN}}^{\max }-C_{\mathrm{NN}}}\right)
$$

where $C_{\mathrm{NN}}^{\min }=-0.1$ and $C_{\mathrm{NN}}^{\max }$ is the maximum value of $C_{\mathrm{NN}}$ obtained from a large sample of signal MC decays. The distribution of $C_{\mathrm{NN}}^{\prime}$ is well modeled by a Gaussian function. Backgrounds arising from other $B_{s}^{0}$ and non- $B_{s}^{0}$ decays were studied using MC simulation and found to be negligible.

After applying all selection criteria, approximately $1.0 \%$ of events have multiple $B_{s}^{0}$ candidates. For these events, we retain the candidate having the smallest value of $\chi^{2}$ obtained from the deviations of the reconstructed $K_{s}$ masses from their nominal values [18]. According to MC simulation, this criterion selects the correct $B_{s}^{0}$ candidate $>99 \%$ of the time.

We measure the signal yield by performing an unbinned extended maximum likelihood fit to the variables $M_{\mathrm{bc}}, \Delta E$, and $C_{\mathrm{NN}}^{\prime}$.

The results of the fit are $29.0_{-7.6}^{+8.5}$ signal events and $1095.0_{-33.4}^{+33.9}$ continuum background events. Projections of the fit are shown in Fig. 3. The branching fraction is calculated via

$$
\mathcal{B}\left(B_{s}^{0} \rightarrow K^{0} \bar{K}^{0}\right)=\frac{Y_{s}}{2 N_{B_{s}^{0} \bar{B}_{s}^{0}}(0.50) \mathcal{B}_{K^{0}}^{2} \varepsilon},
$$

where $Y_{s}$ is the fitted signal yield; $N_{B_{s}^{0} \bar{B}_{s}^{0}}=(6.53 \pm 0.66) \times 10^{6}$ is the number of $B_{s}^{0} \overline{B_{s}^{0}}$ events; $\mathcal{B}_{K^{0}}=$ $(69.20 \pm 0.05) \%$ is the branching fraction for $K_{s} \rightarrow \pi^{+} \pi^{-}[18]$; and $\varepsilon=(46.3 \pm 0.1) \%$ is the signal efficiency as determined from MC simulation. The factor 0.50 accounts for the $50 \%$ probability for $K^{0} \bar{K}^{0} \rightarrow K_{s} K_{s}$ (since $K^{0} \bar{K}^{0}$ is $C P$ even). Inserting these values gives $\mathcal{B}\left(B_{s}^{0} \rightarrow K^{0} \bar{K}^{0}\right)=\left(19.6_{-5.1}^{+5.8} \pm\right.$ $1.0 \pm 2.0) \times 10^{-6}$, where the first uncertainty is statistical, the second is systematic, and the third reflects the uncertainty due to the total number of $B_{s}^{0} \bar{B}_{s}^{0}$ pairs. This value is in good agreement with the SM predictions [20], and it implies that the Belle II experiment [26] will reconstruct over 1000 of these decays. Such a sample would allow for a much higher sensitivity search for new physics in this $b \rightarrow s$ penguin-dominated decay.

The signal significance is calculated as $\sqrt{-2 \ln \left(\mathcal{L}_{0} / \mathcal{L}_{\max }\right)}$, where $\mathcal{L}_{0}$ is the likelihood value when the signal yield is fixed to zero, and $\mathcal{L}_{\max }$ is the likelihood value of the nominal fit. We include systematic uncertainties in the significance by convolving the likelihood function with a Gaussian function whose width is equal to that part of the systematic uncertainty that affects the signal yield. We obtain a signal significance of 5.1 standard deviations; thus, our measurement constitutes the first observation of this decay.

\section{Study of $e^{+} e^{-} \rightarrow B^{(*)} \bar{B}^{(*)} \pi^{ \pm}$at $\sqrt{s}=10.866 \mathbf{~ G e V}$}

Two new charged bottomonium-like resonances, $Z_{b}(10610)$ and $Z_{b}(10650)$, have been observed recently by the Belle Collaboration in $e^{+} e^{-} \rightarrow \Upsilon(n \mathrm{~S}) \pi^{+} \pi^{-}, n=1,2,3$ and $e^{+} e^{-} \rightarrow h_{b}(m \mathrm{P}) \pi^{+} \pi^{-}$, $m=1,2[28,29]$. Analysis of the quark composition of the initial and final states reveals that these hadronic objects have an exotic nature: $Z_{b}$ should be comprised of (at least) four quarks including a $b \bar{b}$ pair. Several models [30] have been proposed to describe the internal structure of these states. In Ref. [31], it was suggested that $Z_{b}(10610)$ and $Z_{b}(10650)$ states might be loosely bound $B \bar{B}^{*}$ and $B^{*} \bar{B}^{*}$ systems, respectively. If so, it is natural to expect the $Z_{b}$ states to decay to final states with $B^{(*)}$ mesons at substantial rates. 

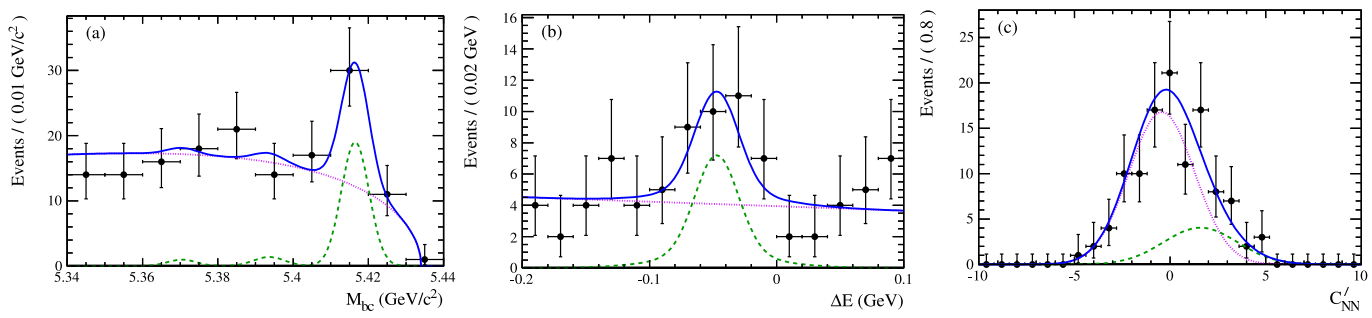

Figure 3. Projections of the $3 \mathrm{D}$ fit to the real data: (a) $M_{\mathrm{bc}}$ in $-0.11 \mathrm{GeV}<\Delta E<0.02 \mathrm{GeV}$ and $C_{\mathrm{NN}}^{\prime}>0.5$; (b) $\Delta E$ in $5.405 \mathrm{GeV} / c^{2}<M_{\mathrm{bc}}<5.427 \mathrm{GeV} / c^{2}$ and $C_{\mathrm{NN}}^{\prime}>0.5$; and (c) $C_{\mathrm{NN}}^{\prime}$ in $5.405 \mathrm{GeV} / c^{2}<M_{\mathrm{bc}}<$ $5.427 \mathrm{GeV} / c^{2}$ and $-0.11 \mathrm{GeV}<\Delta E<0.02 \mathrm{GeV}$. The points with error bars are data, the (green) dashed curves show the signal, (magenta) dotted curves show the continuum background, and (blue) solid curves show the total. The $\chi^{2} /$ (number of bins) values of these fit projections are $0.30,0.43$, and 0.26 , respectively, which indicate that the fit gives a good description of the data. The three peaks in $M_{\mathrm{bc}}$ arise from $\Upsilon(5 S) \rightarrow B_{s}^{0} \bar{B}_{s}^{0}, B_{s}^{* 0} \bar{B}_{s}^{0}+B_{s}^{0} \bar{B}_{s}^{* 0}$, and $B_{s}^{* 0} B_{s}^{0} B_{s}^{* 0}$ decays.

Evidence for the three-body $\Upsilon(10860) \rightarrow B \bar{B}^{*} \pi$ decay has been reported previously by Belle, based on a data sample of $23.6 \mathrm{fb}^{-1}$ [32]. In the analysis [27] Belle uses a data sample with an integrated luminosity of $121.4 \mathrm{fb}^{-1}$ collected near the peak of the $\Upsilon(10860)$ resonance $(\sqrt{s}=10.866 \mathrm{GeV})$ with the Belle detector. Note that we reconstruct only three-body $B^{(*)} \bar{B}^{(*)} \pi$ combinations with a charged primary pion. For brevity, we adopt the following notations: the set of $B^{+} \bar{B}^{0} \pi^{-}$and $B^{-} B^{0} \pi^{+}$ final states is referred to as $B B \pi$; the set of $B^{+} \bar{B}^{* 0} \pi^{-}, B^{-} B^{* 0} \pi^{+}, B^{0} B^{*-} \pi^{+}$and $\bar{B}^{0} B^{*+} \pi^{-}$final states is referred to as $B B^{*} \pi$; and the set of $B^{*+} \bar{B}^{* 0} \pi^{-}$and $B^{*-} B^{* 0} \pi^{+}$final states is denoted as $B^{*} B^{*} \pi$.

$B$ mesons are reconstructed in the following decay channels: $B^{+} \rightarrow J / \psi K^{(*)+}, B^{+} \rightarrow \bar{D}^{(*) 0} \pi^{+}$, $B^{0} \rightarrow J / \psi K^{(*) 0}, B^{0} \rightarrow D^{(*)-} \pi^{+}$(eighteen in total). The dominant background comes from $e^{+} e^{-} \rightarrow c \bar{c}$ continuum events, where true $D$ mesons produced in $e^{+} e^{-}$annihilation are combined with random particles to form a $B$ candidate. This type of background is suppressed using variables that characterize the event topology. We identify $B$ candidates by their reconstructed invariant mass $M(B)$ and momentum $P(B)$ in the center-of-mass (c.m.) frame. We require $P(B)<1.35 \mathrm{GeV} / c$ to retain $B$ mesons produced in both two-body and multibody processes. The $M(B)$ distribution for $B$ candidates is shown in Fig. 4(a). We perform a binned maximum likelihood fit of the $M(B)$ distribution to the sum of a signal component parameterized by a Gaussian function and two background components: one related to other decay modes of $B$ mesons and one due to continuum $e^{+} e^{-} \rightarrow q \bar{q}$ processes, where $q=u, d, s, c$. We find $12263 \pm 168$ fully reconstructed $B$ mesons. The $B$ signal region is defined by requiring $M(B)$ to be within 30 to $40 \mathrm{MeV} / c^{2}$ (depending on the $B$ decay mode) of the nominal $B$ mass.

Reconstructed $B^{+}$or $\bar{B}^{0}$ candidates are combined with $\pi^{-}$'s — the right-sign (RS) combination — and the missing mass, $M_{\text {miss }}(B \pi)$, is calculated as $M_{\text {miss }}(B \pi)=\sqrt{\left(\sqrt{s}-E_{B \pi}\right)^{2} / c^{4}-P_{B \pi}^{2} / c^{2}}$, where $E_{B \pi}$ and $P_{B \pi}$ are the measured energy and momentum of the reconstructed $B \pi$ combination. Signal $e^{+} e^{-} \rightarrow B B^{*} \pi$ events produce a narrow peak in the $M_{\text {miss }}(B \pi)$ spectrum around the nominal $B^{*}$ mass while $e^{+} e^{-} \rightarrow B^{*} B^{*} \pi$ events produce a peak at $m_{B^{*}}+\Delta m_{B^{*}}$, where $\Delta m_{B^{*}}=m_{B^{*}}-m_{B}$, due to the missed photon from the $B^{*} \rightarrow B \gamma$ decay. It is important to note here that, according to signal $\mathrm{MC}, B B^{*} \pi$ events, where the reconstructed $B$ is the one from the $B^{*}$, produce a peak in the $M_{\text {miss }}(B \pi)$ distribution at virtually the same position as $B B^{*} \pi$ events, where the reconstructed $B$ is the primary one. To remove the correlation between $M_{\text {miss }}(B \pi)$ and $M(B)$ and to improve the resolution, we use $M_{\text {miss }}^{*}=$ $M_{\text {miss }}(B \pi)+M(B)-m_{B}$ instead of $M_{\text {miss }}(B \pi)$. The $M_{\text {miss }}^{*}$ distribution for the RS combinations is shown 

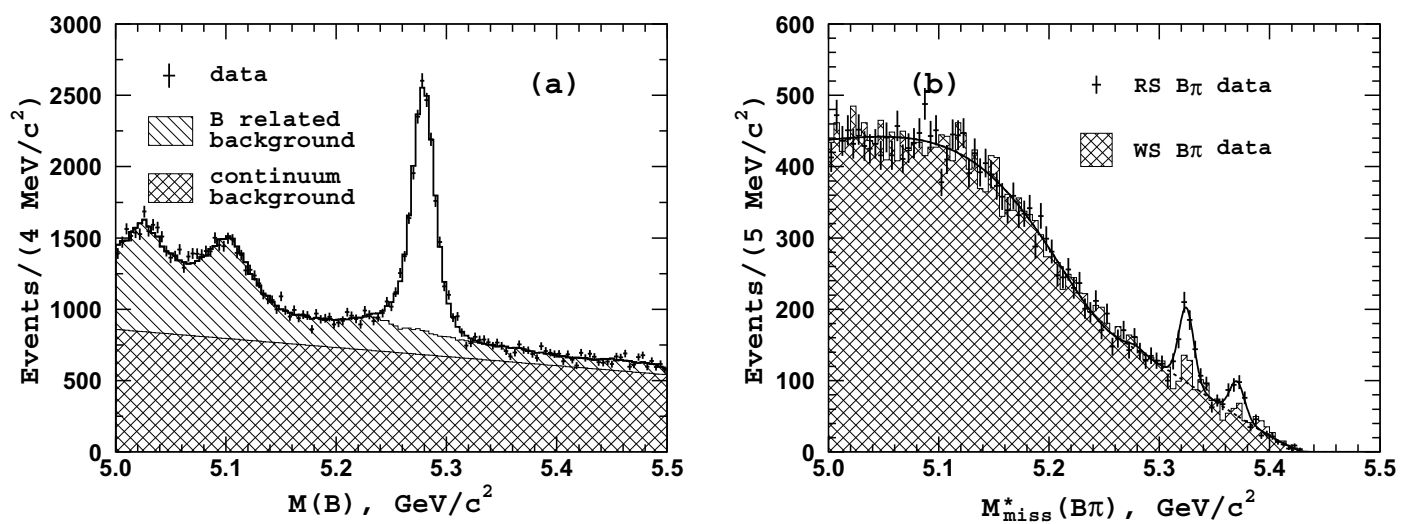

Figure 4. The (a) invariant mass and (b) $M_{\text {miss }}^{*}(B \pi)$ distribution for $B$ candidates in the $B$ signal region. Points with error bars represent the data. The open histogram in (a) shows the result of the fit to data. The solid line in (b) shows the result of the fit to the RS $B \pi$ data; the dashed line represents the background level.

in Fig. 4(b), where peaks corresponding to the $B B^{*} \pi$ and $B^{*} B^{*} \pi$ signals are evident. Combinations with $\pi^{+}$— the wrong sign (WS) combinations - are used to evaluate the shape of the combinatorial background. There is also a hint for a peaking structure in the WS $M_{\text {miss }}^{*}$ distribution, shown as a hatched histogram in Fig. 4(b). Due to $B^{0}-B^{0}$ oscillations, we expect a fraction of the produced $B^{0}$ mesons to decay as $B^{0}$ given by $0.5 x_{d}^{2} /\left(1+x_{d}^{2}\right)=0.1861 \pm 0.0024$, where $x_{d}$ is the $B^{0}$ mixing parameter [18].

A binned maximum likelihood fit is performed to fit the $M_{\text {miss }}^{*}$ distribution. ISR events produce an $M_{\text {miss }}^{*}$ distribution similar to that for $q \bar{q}$ events; these two components are modeled by a single threshold function. The resolution of the signal peaks in Fig. 4(c) is dominated by the c.m. energy spread and is fixed at $6.5 \mathrm{MeV} / \mathrm{c}^{2}$ as determined from the signal MC. The fit to the RS spectrum yields $N_{B B \pi}=13 \pm 25, N_{B B^{*} \pi}=357 \pm 30$ and $N_{B^{*} B^{*} \pi}=161 \pm 21$ signal events. The statistical significance of the observed $B B^{*} \pi$ and $B^{*} B^{*} \pi$ signal is $9.3 \sigma$ and $8.1 \sigma$, respectively. The statistical significance is calculated as $\sqrt{-2 \ln \left(\mathcal{L}_{0} / \mathcal{L}_{\text {sig }}\right)}$, where $\mathcal{L}_{\text {sig }}$ and $\mathcal{L}_{0}$ denote the likelihood values obtained with the nominal fit and with the signal yield fixed at zero, respectively.

For the subsequent analysis, we require $\left|M_{\text {miss }}^{*}-m_{B^{*}}\right|<15 \mathrm{MeV} / c^{2}$ to select $B B^{*} \pi$ signal events and $\left|M_{\text {miss }}^{*}-\left(m_{B^{*}}+\Delta m_{B}\right)\right|<12 \mathrm{MeV} / c^{2}$, where $\Delta m_{B}=m_{B^{*}}-m_{B}$, to select $B^{*} B^{*} \pi$ events. For the selected $B^{*} B^{(*)} \pi$ candidates, we calculate $M_{\text {miss }}(\pi)=\sqrt{\left(\sqrt{s}-E_{\pi}\right)^{2} / c^{4}-P_{\pi}^{2} / c^{2}}$, where $E_{\pi}$ and $P_{\pi}$ are the reconstructed energy and momentum, respectively, of the charged pion in the c.m. frame. We perform a simultaneous binned maximum likelihood fit to the RS and WS samples, assuming the same number and distribution of background events in both samples and known fraction of signal events in the RS sample that leaks to the WS sample due to mixing. To fit the $M_{\text {miss }}(\pi)$ spectrum, we use the function

$$
F(m)=\left[f_{\mathrm{sig}} S(m)+B(m)\right] \epsilon(m) F_{\mathrm{PHSP}}(m),
$$

where $m \equiv M_{\text {miss }}(\pi) ; f_{\text {sig }}=1.0(0.1105 \pm 0.0016$, [33]) for the RS (WS) sample; $S(m)$ and $B(m)$ are the signal and background PDFs, respectively; and $F_{\mathrm{PHSP}}(m)$ is the phase space function. To account for the instrumental resolution, we smear the function $F(m)$ with a Gaussian function. 
Table 2. Summary of fit results to the $M_{\text {miss }}(\pi)$ distributions for the three-body $B B^{*} \pi$ and $B^{*} B^{*} \pi$ final states.

\begin{tabular}{|c|c|c|c|c|c|c|c|}
\hline \multirow[t]{2}{*}{ Mode } & \multirow[t]{2}{*}{ Parameter } & \multirow[t]{2}{*}{ Model-0 } & \multicolumn{2}{|c|}{$\overline{\overline{\text { Model-1 }}}$} & \multicolumn{2}{|c|}{$\overline{\overline{\text { Model-2 }}}$} & \multirow[t]{2}{*}{ Model-3 } \\
\hline & & & Solution 1 & Solution 2 & Solution 1 & Solution 2 & \\
\hline \multirow[t]{6}{*}{$B B^{*} \pi$} & $f_{Z_{b}(10610)}$ & 1.0 & $1.45 \pm 0.24$ & $0.64 \pm 0.15$ & $1.01 \pm 0.13$ & $1.18 \pm 0.15$ & - \\
\hline & $f_{Z_{b}(10650)}$ & - & - & - & $0.05 \pm 0.04$ & $0.24 \pm 0.11$ & - \\
\hline & $\phi_{Z_{b}(10650)}$, rad. & - & - & - & $-0.26 \pm 0.68$ & $-1.63 \pm 0.14$ & - \\
\hline & $f_{\mathrm{nr}}$ & - & $0.48 \pm 0.23$ & $0.41 \pm 0.17$ & - & - & 1.0 \\
\hline & $\phi_{\mathrm{nr}}, \mathrm{rad}$ & - & $-1.21 \pm 0.19$ & $0.95 \pm 0.32$ & - & - & - \\
\hline & $-2 \log \mathcal{L}$ & -304.7 & -300.6 & -300.5 & -301.4 & -301.4 & -344.5 \\
\hline \multirow[t]{4}{*}{$B^{*} B^{*} \pi$} & $f_{Z_{b}(10650)}$ & 1.0 & $1.04 \pm 0.15$ & $0.77 \pm 0.22$ & & & - \\
\hline & $f_{\mathrm{nr}}$ & - & $0.02 \pm 0.04$ & $0.24 \pm 0.18$ & & & 1.0 \\
\hline & $\phi_{\mathrm{nr}}, \mathrm{rad}$ & - & $0.29 \pm 1.01$ & $1.10 \pm 0.44$ & & & - \\
\hline & $-2 \log \mathcal{L}$ & -182.4 & -182.4 & -182.4 & & & -209.7 \\
\hline
\end{tabular}

We first analyze of the $B B^{*} \pi\left[B^{*} B^{*} \pi\right]$ data with the simplest hypothesis, Model- 0 , that includes only the $Z_{b}(10610)\left[Z_{b}(10650)\right]$ amplitude. Results of the fit are shown in Fig. 5; the numerical results are summarized in Table 2. The fraction $f_{X}$ of the total three-body signal attributed to a particular quasi-two-body intermediate state is calculated as $f_{X}=\int\left|\mathcal{A}_{X}\right|^{2} d m / \int S(m) d m$, where $\mathcal{A}_{X}$ is the amplitude for a particular component $X$ of the three-body amplitude. Next, we extend the hypothesis to include a possible non-resonant component, Model-1, and then the $B B^{*} \pi$ data is fit to a combination of two $Z_{b}$ amplitudes, Model-2. In both cases, we do not get a statistically significant improvement in the data description: the likelihood value is only marginally improved (see Table 2). The addition of extra components to the amplitude also produces multiple maxima in the likelihood function. As a result, we use Model-0 as our nominal hypothesis. Finally, we fit both samples to a pure non-resonant amplitude (Model-3). In this case, the fit is significantly worse.

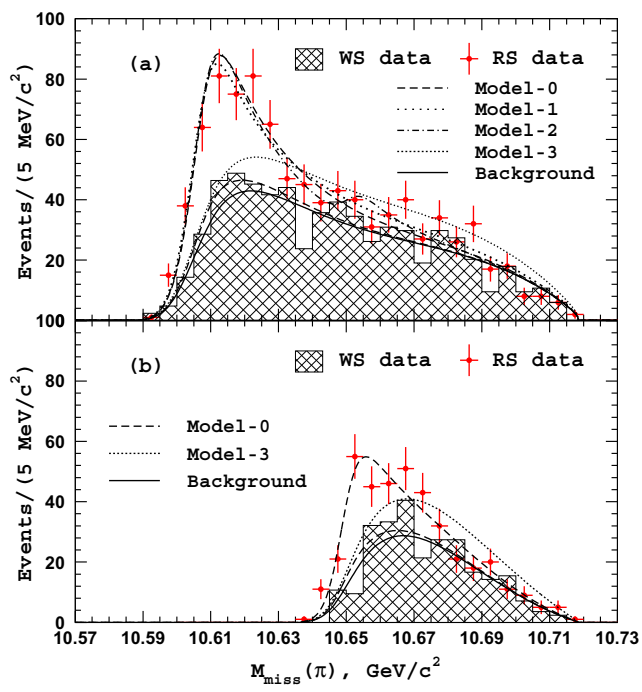

Figure 5. The $M_{\text {miss }}(\pi)$ distribution for the (a) $B B^{*} \pi$ and (b) $B^{*} B^{*} \pi$ candidate events.

If the parameters of the $Z_{b}$ resonances are allowed to float, the fit to the $B B^{*} \pi$ data with Model-0 gives $10605 \pm 6 \mathrm{MeV} / c^{2}$ and $25 \pm 7 \mathrm{MeV}$ for the $Z_{b}(10610)$ mass and width, respectively, and the 
Table 3. $B$ branching fractions for the $Z_{b}^{+}(10610)$ and $Z_{b}^{+}(10650)$ decays. The first quoted uncertainty is statistical; the second is systematic.

\begin{tabular}{lcc}
\hline \hline Channel & \multicolumn{2}{c}{ Fraction, \% } \\
& $Z_{b}(10610)$ & $Z_{b}(10650)$ \\
\hline$\Upsilon(1 \mathrm{~S}) \pi^{+}$ & $0.60 \pm 0.17 \pm 0.07$ & $0.17 \pm 0.06 \pm 0.02$ \\
$\Upsilon(2 \mathrm{~S}) \pi^{+}$ & $4.05 \pm 0.81 \pm 0.58$ & $1.38 \pm 0.45 \pm 0.21$ \\
$\Upsilon(3 \mathrm{~S}) \pi^{+}$ & $2.40 \pm 0.58 \pm 0.36$ & $1.62 \pm 0.50 \pm 0.24$ \\
$h_{b}(1 \mathrm{P}) \pi^{+}$ & $4.26 \pm 1.28 \pm 1.10$ & $9.23 \pm 2.88 \pm 2.28$ \\
$h_{b}(2 \mathrm{P}) \pi^{+}$ & $6.08 \pm 2.15 \pm 1.63$ & $17.0 \pm 3.74 \pm 4.1$ \\
$B^{+} \bar{B}^{* 0}+\bar{B}^{0} B^{*+}$ & $82.6 \pm 2.9 \pm 2.3$ & - \\
$B^{*+} \bar{B}^{* 0}$ & - & $70.6 \pm 4.9 \pm 4.4$ \\
\hline \hline
\end{tabular}

fit to the $B^{*} B^{*} \pi$ data gives $10648 \pm 13 \mathrm{MeV} / c^{2}$ and $23 \pm 8 \mathrm{MeV}$ for the $Z_{b}(10650)$ mass and width, respectively. The large errors here reflect the strong correlation between the resonance parameters.

Using the results of the fit to the $M_{\text {miss }}(\pi)$ spectra with the nominal model (Model-0 in Table 2) and the results of the analyses of $e^{+} e^{-} \rightarrow \Upsilon(n \mathrm{~S}) \pi^{+} \pi^{-}[28]$ and $e^{+} e^{-} \rightarrow h_{b}(m \mathrm{P}) \pi^{+} \pi^{-}[34,35]$, we calculate the ratio of the branching fractions $\mathcal{B}\left(Z_{b}(10610) \rightarrow B \bar{B}^{*}+\right.$ c.c. $) / \mathcal{B}\left(Z_{b}(10610) \rightarrow\right.$ bottomonium $)=$ $4.76 \pm 0.64 \pm 0.75$ and $\mathcal{B}\left(Z_{b}(10650) \rightarrow B^{*} \bar{B}^{*}\right) / \mathcal{B}\left(Z_{b}(10650) \rightarrow\right.$ bottomonium $)=2.40 \pm 0.44 \pm 0.50$.

We calculate the relative fractions for $Z_{b}$ decays, assuming that they are saturated by the already observed $\Upsilon(n \mathrm{~S}) \pi, h_{b}(m \mathrm{P}) \pi$, and $B^{*} B^{(*)}$ channels. The results are summarized in Table 3.

In conclusion, we report the first observations of the three-body $e^{+} e^{-} \rightarrow B B^{*} \pi$ and $e^{+} e^{-} \rightarrow B^{*} B^{*} \pi$ processes with a statistical significance above $8 \sigma$. The analysis of the $B^{(*)} B^{*}$ mass spectra indicates that the total three-body rates are dominated by the intermediate $e^{+} e^{-} \rightarrow Z_{b}(10610)^{\mp} \pi^{ \pm}$and $e^{+} e^{-} \rightarrow$ $Z_{b}(10650)^{\mp} \pi^{ \pm}$transitions for the $B B^{*} \pi$ and $B^{*} B^{*} \pi$ final states, respectively.

\section{Observation of the decay $\Lambda_{c}^{+} \rightarrow p K^{+} \pi^{-}$}

Several doubly Cabibbo-suppressed (DCS) decays of charmed mesons have been observed. Their measured branching ratios with respect to corresponding Cabibbo-favored (CF) decays play an important role in constraining models of the decay of charmed hadrons and in the study of flavor- $S U(3)$ symmetry [37]. Because of the smaller production cross sections for charmed baryons, DCS decays of charmed baryons have not yet been observed, and only an upper limit, $\mathcal{B}\left(\Lambda_{c}^{+} \rightarrow p K^{+} \pi^{-}\right) / \mathcal{B}\left(\Lambda_{c}^{+} \rightarrow\right.$ $\left.p K^{-} \pi^{+}\right)<0.46 \%$ with $90 \%$ confidence level, has been reported by the FOCUS Collaboration [38]. Theoretical calculations of DCS decays of charmed baryons have been limited to two-body decay modes [39, 40].

Recently Belle reported the first observation of the DCS decay $\Lambda_{c}^{+} \rightarrow p K^{+} \pi^{-}$and the measurement of its branching ratio with respect to its counterpart CF decay $\Lambda_{c}^{+} \rightarrow p K^{-} \pi^{+}$. In the letter [36], Belle reports the first observation of the DCS decay $\Lambda_{c}^{+} \rightarrow p K^{+} \pi^{-}$and the measurement of its branching ratio with respect to its counterpart $\mathrm{CF}$ decay $\Lambda_{c}^{+} \rightarrow p K^{-} \pi^{+}$. Unlike charmed meson decays, internal $W$ emission and $W$ exchange are not suppressed for charmed baryon decays. In previous studies of CF or singly Cabibbo-suppressed (SCS) decays of $\Lambda_{c}^{+}$and $\Xi_{c}^{0}$, direct evidence of $W$ exchange and internal $W$ emission has been observed [41]. When we consider that $W$ exchange is prohibited in $\Lambda_{c}^{+} \rightarrow p K^{+} \pi^{-}$but allowed in $\Lambda_{c}^{+} \rightarrow p K^{-} \pi^{+}$, the contribution of $W$ exchange to $\Lambda_{c}^{+}$decays can be estimated by comparing the measured branching ratio with the naïve expectation [38], $\tan ^{4} \theta_{\mathrm{c}}$ $(0.285 \%)$, where $\theta_{\mathrm{c}}$ is the Cabibbo mixing angle [42] and $\sin \theta_{\mathrm{c}}=0.225 \pm 0.001$ [18]. This approach does not take into account effects of flavor- $S U(3)$ symmetry breaking. 
A $\Lambda_{c}^{+}$candidate is reconstructed from the three charged hadrons. To suppress combinatorial backgrounds, especially from $B$ meson decays, we place a requirement on the scaled momentum: $x_{p}>0.53$, where $x_{p}$ is defined as $p^{*} / \sqrt{E_{\mathrm{cm}}^{2} / 4-M^{2}}$; here, $E_{\mathrm{cm}}$ is the total center-of-mass energy, $p^{*}$ is the momentum in the center-of-mass frame, and $M$ is the mass of the $\Lambda_{c}^{+}$candidate. In addition, the $\chi^{2}$ value from the common vertex fit of the charged tracks must be less than 40 .
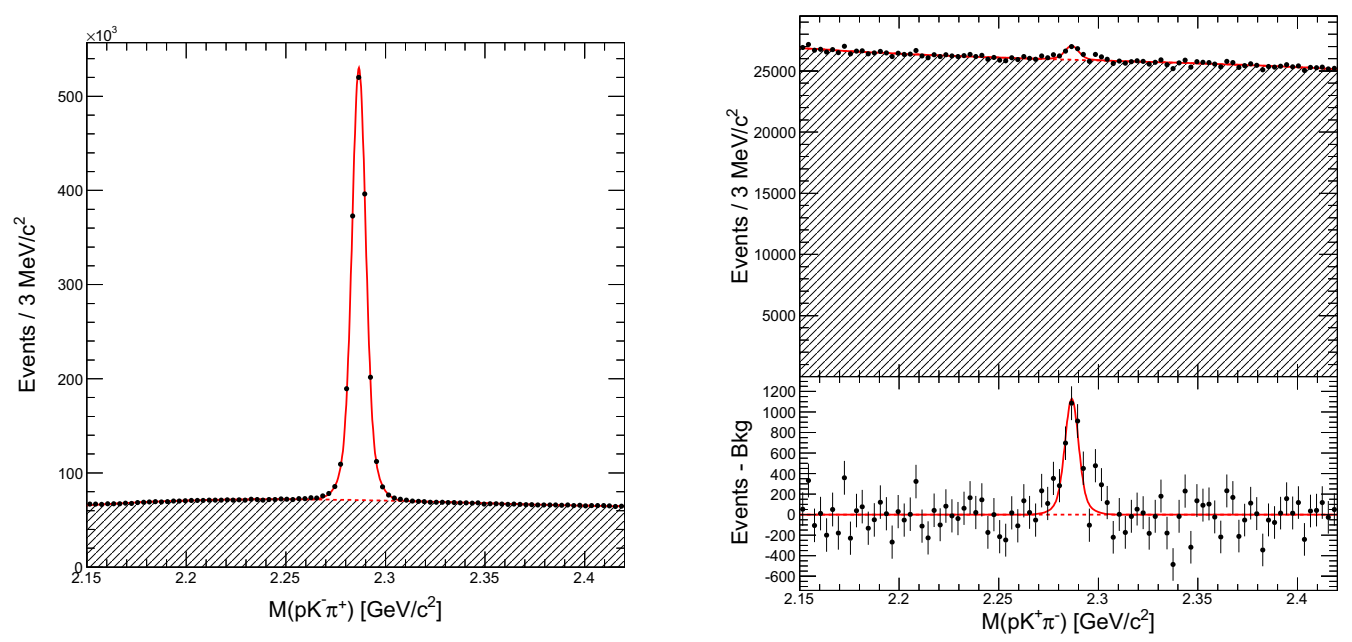

Figure 6. Distribution of $M\left(p K^{-} \pi^{+}\right)$(left), $M\left(p K^{+} \pi^{-}\right)$(top right) and residuals of data with respect to the fitted combinatorial background (bottom right). The curves indicate the fit result: the full fit mode 1 (solid) and the combinatoric background only (dashed).

Figures 6,a) and 6,b) show invariant mass distributions, $M\left(p K^{-} \pi^{+}\right)(\mathrm{CF})$ and $M\left(p K^{+} \pi^{-}\right)(\mathrm{DCS})$, with the final selection criteria. DCS decay events are clearly observed in $M\left(p K^{+} \pi^{-}\right)$. We perform a binned least- $\chi^{2}$ fit to the two distributions from $2.15 \mathrm{GeV} / c^{2}$ to $2.42 \mathrm{GeV} / c^{2}$ with $0.01 \mathrm{MeV} / c^{2}$ bin width, and the figures are drawn with merged bins. The DCS decay has a peaking background from the SCS decay $\Lambda_{c}^{+} \rightarrow \Lambda K^{+}$with $\Lambda \rightarrow p \pi^{-}$, which has the same final state topology. However, because of the long $\Lambda$ lifetime, many of the $\Lambda$ vertexes are displaced by several centimeters from the main vertex so the geometrical requirements suppress most of this background. The remaining SCS-decay yield is included in the signal yield of $\Lambda_{c}^{+} \rightarrow p K^{+} \pi^{-}$decay and is estimated via the relation

$$
\mathcal{N}\left(S C S ; \Lambda \rightarrow p \pi^{-}\right)=\frac{\epsilon\left(S C S ; \Lambda \rightarrow p \pi^{-}\right)}{\epsilon(C F)} \frac{\mathcal{B}\left(S C S ; \Lambda \rightarrow p \pi^{-}\right)}{\mathcal{B}(C F)} \mathcal{N}(C F),
$$

where $\mathcal{N}(C F)$ is the signal yield of the CF decay, $\mathcal{B}\left(S C S ; \Lambda \rightarrow p \pi^{-}\right) / \mathcal{B}(C F)=(0.61 \pm 0.13) \%$ is the branching ratio [18], and $\epsilon\left(S C S ; \Lambda \rightarrow p \pi^{-}\right) / \epsilon(C F)=0.023$ is the relative efficiency found using MC samples. After subtraction of this SCS component, the signal yield of the DCS decay is $3379 \pm 380 \pm 78$, where the first uncertainty is statistical and the second is systematic due to this subtraction. To estimate the statistical significance of the DCS signal, we exclude the SCS signal by vetoing events with $1.1127 \mathrm{GeV} / c^{2}<M\left(p \pi^{-}\right)<1.1187 \mathrm{GeV} / c^{2}$. The significance is estimated as $\sqrt{-2 \ln \left(\mathcal{L}_{0} / \mathcal{L}\right)}$, where $\mathcal{L}_{0}$ and $\mathcal{L}$ are the maximum likelihood values from binned maximum likelihood fits with the signal yield fixed to zero and allowed to float, respectively. The calculated significance corresponds to $9.4 \sigma$. 
The branching ratio, $\mathcal{B}\left(\Lambda_{c}^{+} \rightarrow p K^{+} \pi^{-}\right) / \mathcal{B}\left(\Lambda_{c}^{+} \rightarrow p K^{-} \pi^{+}\right)$, is $(2.35 \pm 0.27 \pm 0.21) \times 10^{-3}$, where the uncertainties are statistical and systematic, respectively. The branching fraction of the CF decay, $\left(6.84 \pm 0.24_{-0.27}^{+0.21}\right) \times 10^{-2}$, was already well-measured in a previous Belle analysis [43]. Combining that with our measurement, we determine the absolute branching fraction of the DCS decay to $\left(1.61 \pm 0.23_{-0.08}^{+0.07}\right) \times 10^{-4}$, where the first uncertainty is the total uncertainty of the branching ratio and the second is uncertainty of the branching fraction of CF decay. This measured branching ratio corresponds to $(0.82 \pm 0.12) \tan ^{4} \theta_{\mathrm{c}}$, where the uncertainty is the total.

\section{References}

[1] A. Abashian et al. (Belle Collab.), Nucl. Instr. and Meth. A 479, 117 (2002).

[2] S. Kurokawa and E. Kikutani, Nucl. Instrum. Methods Phys. Res., Sect. A 499, 1 (2003), and other papers included in this volume; T. Abe et al., Prog. Theor. Exp. Phys. (2013) 03A001 and following articles up to 03A011.

[3] A. Abdesselam et al. (Belle Collaboration), arXiv:1604.04042.

[4] R. Aaij et al. JHEP 02, 104 (2016).

[5] R. Aaij et al. JHEP 08, 131 (2013).

[6] S. Descotes-Genon, J. Matias, M. Ramon, and J. Virto, JHEP 01, 048 (2013).

[7] S. Descotes-Genon, T. Hurth, J. Matias, and J. Virto. JHEP 1305, 137 (2013).

[8] R. Aaij et al. Phys. Rev. Lett. 111, 191801 (2013).

[9] S. Fajfer, J.F.Kamenik, and I. Nisandzic, Phys. Rev. D 85, 094025 (2012).

[10] J.F. Kamenik, and F. Mescia, Phys. Rev. D 78, 014003 (2008).

[11] J.P. Lees et al. (BABAR Collaboration), Phys. Rev. Lett. 109, 101802 (2012); J.P. Lees et al. (BABAR Collaboration), Phys. Rev. D 88, 072012 (2013);

[12] A. Matyja et al. (Belle Collaboration), Phys. Rev. Lett. 99, 191807 (2007).

[13] A. Bozek et al. (Belle Collaboration), Phys. Rev. D 82, 072005 (2010).

[14] M. Huschle et al. (Belle Collaboration), Phys. Rev. D 92, 072014 (2015).

[15] R. Aaij et al. (LHCb Collaboration), Phys. Rev. Lett. 115, 111803 (2015).

[16] Y. Amhis et al., arXiv:1412.7515 and online update at http://www.slac.stanford.edu/xorg/hfag/

[17] A. Abdesselam et al. (Belle collaboration), arXiv:1603.06711.

[18] K. A. Olive et al. (Particle Data Group), Chin. Phys. C 38, 090001 (2014).

[19] B. Pal et al. (Belle Collaboration), Phys. Rev. Lett. 116, 161801 (2016).

[20] C. H. Chen, Phys. Lett. B 520, 33 (2001); A. R. Williamson and J. Zupan, Phys. Rev. D 74, 014003 (2006); A. Ali et al., Phys. Rev. D 76, 074018 (2007); C. K. Chua, Phys. Rev. D 78, 076002 (2008); K. Wang and G. Zhu, Phys. Rev. D 88, 014043 (2013); J. J. Wang, D. T. Lin, W. Sun, Z. J. Ji, S. Cheng and Z. J. Xiao, Phys. Rev. D 89, 074046 (2014); Q. Chang, J. Sun, Y. Yang and X. Li, Phys. Lett. B 740, 56 (2015); H. Y. Cheng, C. W. Chiang and A. L. Kuo, Phys. Rev. D 91, 014011 (2015).

[21] Q. Chang, X. Q. Li and Y. D. Yang, J. Phys. G 41, 105002 (2014).

[22] S. Baek, D. London, J. Matias and J. Virto, J. High Energy Phys. 12, (2006) 019; A. Hayakawa, Y. Shimizu, M. Tanimoto and K. Yamamoto, Prog. Theor. Exp. Phys. 2014, $023 B 04$ (2014).

[23] C.-C. Peng et al. (Belle Collaboration), Phys. Rev. D 82, 072007 (2010).

[24] C. Oswald et al. (Belle Collaboration), Phys. Rev. D 92, 072013 (2015)

[25] S. Esen et al. (Belle Collaboration), Phys. Rev. D 87, 031101(R) (2013).

[26] T. Abe et al. (Belle II Collaboration), arXiv:1011.0352.

[27] A. Garmash et al. (Belle Collaboration), Phys. Rev. Lett. 116, 212001 (2016). 
[28] A. Bondar et al. (Belle Collaboration), Phys. Rev. Lett. 108, 122001 (2012).

[29] A. Garmash et al. (Belle Collaboration), Phys. Rev. D 91, 072003 (2015).

[30] D.-Y. Chen and X. Liu, Phys. Rev. D 84, 094003 (2011); A. Ali, C. Hambrock and W. Wang, Phys. Rev. D 85, 054011 (2012); I. V. Danilkin, V. D. Orlovsky and Y. A. Simonov, Phys. Rev. D 85, 034012 (2012); S. Ohkoda, Y. Yamaguchi, S. Yasui, K. Sudoh and A. Hosaka, Phys. Rev. D 86, 014004 (2012); E. Braaten, C. Langmack and D. Hudson Smith, Phys. Rev. D 90, 014044 (2014).

[31] A. Bondar, A. Garmash, A.I. Milstein, R. Mizuk, and M.B. Voloshin, Phys. Rev. D 84, 054010 (2011).

[32] A. Drutskoy et al. (Belle Collaboration), Phys. Rev. D 81, 112003 (2010).

[33] Determined using mixing parameter $x_{d}$ and the ratio of charged to neutral $B$ yields measured in data from two-body $e^{+} e^{-} \rightarrow B^{(*)} B^{(*)}$ processes.

[34] I. Adachi et al. (Belle Collaboration), Phys. Rev. Lett. 108, 032001 (2012).

[35] The fits to the $M_{\text {miss }}(\pi)$ distributions performed in Ref. [28] give fractions of $f_{Z(10610)^{ \pm} \pi^{\mp}}=$ $0.423_{-0.127-0.009}^{+0.05+0.007}\left(0.352_{-0.004-0.134}^{+0.156+0.001}\right)$ and $f_{Z(10650)^{ \pm} \pi^{\mp}}=0.602_{-0.211-0.038}^{+0.103+0.11}\left(0.648_{-0.114-0.155}^{+0.152+0.067}\right)$ for the $h_{b}(1 \mathrm{P})\left(h_{b}(2 \mathrm{P})\right)$ decays.

[36] S.B. Yang et al. (Belle collaboration), Phys. Rev. Lett. 117, 011801 (2016).

[37] B.R. Ko et al. (Belle Collaboration), Phys. Rev. Lett. 102, 221802 (2009).

[38] J.M. Link et al. (FOCUS Collaboration), Phys. Lett. B 624, 166 (2005).

[39] K.K. Sharma and R.C. Verma, Phys. Rev. D 55, 7067 (1997).

[40] T. Uppal, R.C. Verma, and M.P. Khanna, Phys. Rev. D 49, 3417 (1994).

[41] S. Henderson et al. (CLEO Collaboration), Phys. Lett. B 283, 161 (1992); P. Avery et al. (CLEO Collaboration), Phys. Rev. Lett. 71, 2391 (1993); K. Abe et al. (Belle Collaboration), Phys. Lett. B 524, 33 (2002); B. Aubert et al. (BABAR Collaboration), Phys. Rev. Lett. 95, 142003 (2005);

R. Chistov et al. (Belle Collaboration), Phys. Rev. D 88, 071103(R) (2013).

[42] N. Cabibbo, Phys. Rev. Lett. 10, 531 (1963).

[43] A. Zupanc et al. (Belle Collaboration), Phys. Rev. Lett. 113, 042002 (2014). 